\title{
Towards novel regularization approaches to PET image reconstruction
}

\author{
E. Karali, D. Koutsouris
}

Department of Electrical and Computer Engineering, National Technical University of Athens, Athens, Greece

Email: ekarali76@hotmail.com

Received August 2013

\begin{abstract}
The purpose of this study is to introduce a novel empirical iterative algorithm for medical image reconstruction, under the short name MRP-ISWLS (Median Root Prior Image Space Weighted Least Squares). Further, we assess the performance of the new algorithm by comparing it to the simultaneous version of known MRP algorithms. All algorithms are compared in terms of cross-correlation and CNRs (Contrast-to-Noise Ratios). As it turns out, MRP-ISWLS presents higher CNRs than the known algorithms for objects of different size. Also MRP-ISWLS has better noise manipulation.
\end{abstract}

Keywords: Image Reconstruction; Positron Emission Tomography (PET); Small Animal Imaging; Median Root Prior (MRP)

\section{INTRODUCTION}

Iterative techniques are divided into two main categories: algebraic and statistical. Statistical techniques are classified to maximum-likelihood algorithms and least squares methods. The most famous maximum likelihood technique is the expectation maximization maximum likelihood (EM-ML) algorithm, which was first presented by Shepp and Vardi. Image Space Reconstruction Algorithm (ISRA) is a least square reconstruction method introduced by Daube-Witherspoon and Muehllehner. It shows better noise manipulation than EM-ML. Another least squares algorithm is the Weighted Least Square technique (WLS), due to Anderson et al. WLS assumes that random independent noise variables present different standard deviations. The matrix of these standard deviations consists of the expected projection data. WLS accelerates the reconstruction process and results in reconstructed images of better spatial resolution [1-3].

For the reduction of the noise many regularization methods have been proposed, which reduce drastically the noise with a small image resolution reduction. These methods take into account a priori information for the radioactivity spatial distribution inside the object under examination [1]. The success of a regularization method depends on the mathematical formula of the prior. Median root prior (MRP) [4] belongs to the most popular priors. It is derived from a Gaussian distribution with mean value the median value of reconstructed image pixels in the vicinity of pixel $i$. The use of MRP results in noise component reduction while at the same time it preserves the edges.

The purpose of this study is on the one hand to introduce a new empirical MRP image reconstruction algorithm, under the short name MRP-ISWLS (Median RootPrior-Image Space Weighted Least Squares). MRPISWLS will be an MRP version of ISWLS (Image Space Weigthed Least Squares), which was introduced in [5]. ISWLS has ISRA properties in noise manipulation and WLS acceleration of reconstruction process. To assess the performance of the new iterative reconstruction method we have used phantom data produced from simulating a prototype small-animal PET system. We compared reconstruction data with MRP-EM-ML, MRPISRA and MRP-WLS following the OSL (One Step Late) philoshophy [6]. The methods presented here are applied to 2D sinograms. Moreover, the simultaneous version of the aforementioned algorithms is implemented.

We note that simultaneous versions of reconstruction algorithms, that is, algorithms where all image pixels are simultaneously updated in every iteration, are of great interest because of their ability to be implemented in parallel computing architectures, which decreases drastically the total reconstruction time [1].

\section{THEORY}

In general, every iterative method relies on the hypothesis that the projection data $\mathbf{y}$ is linearly connected to the image $\mathbf{x}$ of radiopharmaceutical spatial distribution, according to the equation:

$$
\mathbf{y}=A^{T} \mathbf{x}
$$

where $A$ is a matrix that characterizes the PET system 
being used for data acquisition. In bibliography this matrix is called system or probability matrix and it projects image data to sinogram domain (the term sinogram refers to the projection data matrix) [1]. Every element $\alpha_{i j}$ of the system matrix $A$ represents the probability an annihilation event emitted in image pixel $i$ to be detected in $\mathrm{LOR}_{\mathrm{j}}$ (Line of Response). The significance of the probability matrix lies on the valuable information related to the data acquisition process, that it can contain (e.g. number of detector rings, number of detector elements in every ring, ring diameter, diameter of transaxial field of view, detector size, image size, spatial and angular sampling).

\subsection{The ISWLS Algorithm}

Consider an image discretized into $N$ pixels and the measured data y collected by $M$ detector tubes. The updating scheme of the ISWLS algorithm in the $k$ th iteration is:

$$
x_{i}^{k}=x_{i}^{k-1} \frac{\sum_{j=1}^{M} a_{i j} y_{j}^{2}}{\sum_{j=1}^{M} a_{i j} \sum_{i^{\prime}=1}^{N}\left(a_{i^{\prime} j} x_{i^{\prime}}^{k-1}\right)^{2}}
$$

\subsection{The Median Root Prior (MRP)}

It is derived from a Gaussian distribution with mean value the median value of reconstructed image pixels in the vicinity of pixel $i$.

Suppose that:

$$
f\left(x_{i}\right) \approx e^{-b \frac{\left(x_{i}-M\right)^{2}}{2 M}}
$$

where $M=\operatorname{med}\left(x_{i}, i\right)$, the median value of reconstructed image pixels in the vicinity of pixel $i$. Then:

$$
u\left(x_{i}\right)=\frac{\partial f\left(x_{i}\right)}{\partial x_{i}}=b \frac{x_{i}-\operatorname{med}\left(x_{i}, i\right)}{\operatorname{med}\left(x_{i}, i\right)}
$$

The term $b \in[0,2]$ determines the degree of smoothing in reconstruction images. If $b=0$ no prior is applied. Big values of $b$ cause over-smoothing, while small values of $b$ result in images with high resolution but with increased noise.

\subsection{MRP Algorithms}

According to the one-step-late philosophy, where the prior is applied to the previous radiopharmaceutical distribution estimation, we can extract an empirical iterative formula for the ISWLS algorithm in combination with MRP prior. The new empirical iterative algorithm has updating scheme:

$$
x_{i}^{k}=\frac{x_{i}^{k-1}}{1+b \frac{x_{i}^{k-1}-\operatorname{med}\left(x_{i}^{k-1}, i\right)}{\operatorname{med}\left(x_{i}^{k-1}, i\right)}} \frac{\sum_{j=1}^{M} a_{i j} y_{j}^{2}}{\sum_{j=1}^{M} a_{i j}\left(\sum_{i^{\prime}=1}^{N} a_{i^{\prime} j^{\prime}} x_{i^{\prime}}^{k-1}\right)^{2}}
$$

The MRP-EM-ML updating scheme in $k$ th iteration is:

$$
x_{i}^{k}=\frac{x_{i}^{k-1}}{1+b \frac{x_{i}^{k-1}-\operatorname{med}\left(x_{i}^{k-1}, i\right)}{\operatorname{med}\left(x_{i}^{k-1}, i\right)}} \sum_{j=1}^{M} \frac{a_{i j} y_{j}}{\left(\sum_{i^{\prime}=1}^{N} a_{i^{\prime} j} x_{i^{\prime}}^{k-1}\right)}
$$

The MRP-ISRA updating scheme in $k$ th iteration is:

$$
x_{i}^{k}=\frac{x_{i}^{k-1}}{1+b \frac{x_{i}^{k-1}-\operatorname{med}\left(x_{i}^{k-1}, i\right)}{\operatorname{med}\left(x_{i}^{k-1}, i\right)} \frac{\sum_{j=1}^{M} a_{i j} y_{j}}{\sum_{j=1}^{M} a_{i j} \sum_{i^{\prime}=1}^{N} a_{i^{\prime} j} x_{i^{\prime}}^{k-1}}}
$$

The MRP-WLS updating scheme in $k$ th iteration is

$$
x_{i}^{k}=\frac{x_{i}^{k-1}}{1+b \frac{x_{i}^{k-1}-\operatorname{med}\left(x_{i}^{k-1}, i\right)}{\operatorname{med}\left(x_{i}^{k-1}, i\right)}} \sum_{j=1}^{M} \frac{a_{i j} y_{j}^{2}}{\left(\sum_{i^{\prime}=1}^{N} a_{i^{\prime} j} x_{i^{\prime}}^{k-1}\right)^{2}}
$$

\section{RESULTS}

For the evaluation of the iterative reconstruction methods presented in Section 2, projection data of a Derenzo-type phantom have been used. The Derenzo-type phantom consists in sets of rods filled with $\mathrm{F}^{18}$, with diameters 4.8, 4, 3.2, 2.4, 1.6, and $1.2 \mathrm{~mm}$, and the same separation between surfaces in the corresponding sets. The rods were surrounded by plastic (polyethylene). Data were produced using Monte Carlo simulation of a small-animal PET scanner, consisting of two detector heads.

Further, $18 \times 10^{6}$ coincidence events were collected. Projection data was binned to a 2D sinogram, 55 pixels $\times$ 170 pixels in size, which means that data from 55 TORs (Tube of Response) per rotation angle were collected and 170 totally angular samples were used. Since the two detector heads rotate from $0^{\circ}$ to $180^{\circ}$ the angular step size was $1.0647^{\circ}$.

The system matrix was derived from an analytical method and calculated once before reconstruction. Each element $a_{i j}$ was computed as the area of intersection $E_{i j}$, of $\mathrm{TOR}_{\mathrm{j}}$ (Tube-of-Response) with image pixel $i$. The calculated system matrix is a sparse matrix. It consists of zero-valued elements in majority that have no contribution during iterative reconstruction process. So, only the non-zero elements were stored, resulting in significant reduction in system matrix size and consequently in required storage. The reconstructed 2D images were 128 pixels $\times 128$ pixels in size, thus the system matrix consisted of $55 \times 170 \times 128 \times 128$ elements with $4.33 \%$ 
sparsity.

The initial image estimate for all MRP algorithms was:

$$
x_{o_{i}}=\frac{\sum_{j=1}^{M} y_{j}}{N}, \quad \mathrm{i}=1,2, \cdots, \mathrm{N}
$$

where $y_{j}$ is the value of the $j^{\text {th }}$ sinogram element and $N$ represents the total number of image pixels ( $N=128 \times$ 128 in this implementation). The value of $b$ was 0.001 .

Figure 1 shows the reconstructed transaxial images with MRP-EMML, MRP-ISRA, MRP-WLS and MRPISWLS after 1, 10 and 50 iterations.

In Figure 2, cross-correlation coefficient $c$ of every iterative method is plotted versus the number of iterations. The cross-correlation coefficient $c$ was calculated according to the equation:

$$
c=\frac{\sum_{i=1}^{N} \sum_{j=1}^{N}\left(\text { Irecon }_{i j}-\overline{\text { Irecon }}\right)\left(\text { Ireal }_{i j}-\overline{\text { Ireal }}\right)}{\sqrt{\sum_{i=1}^{N} \sum_{j=1}^{N}\left(\text { Irecon }_{i j}-\overline{\text { Irecon }}^{2} \sum_{i=1}^{N} \sum_{j=1}^{N}\left(\text { Ireal }_{i j}-\overline{\text { Ireal }}^{2}\right.\right.}}
$$

where $\bar{I}$ recon and $\bar{I}$ real are the reconstructed image and the true phantom activity image mean values, respectively. Cross-correlation coefficient is a similarity
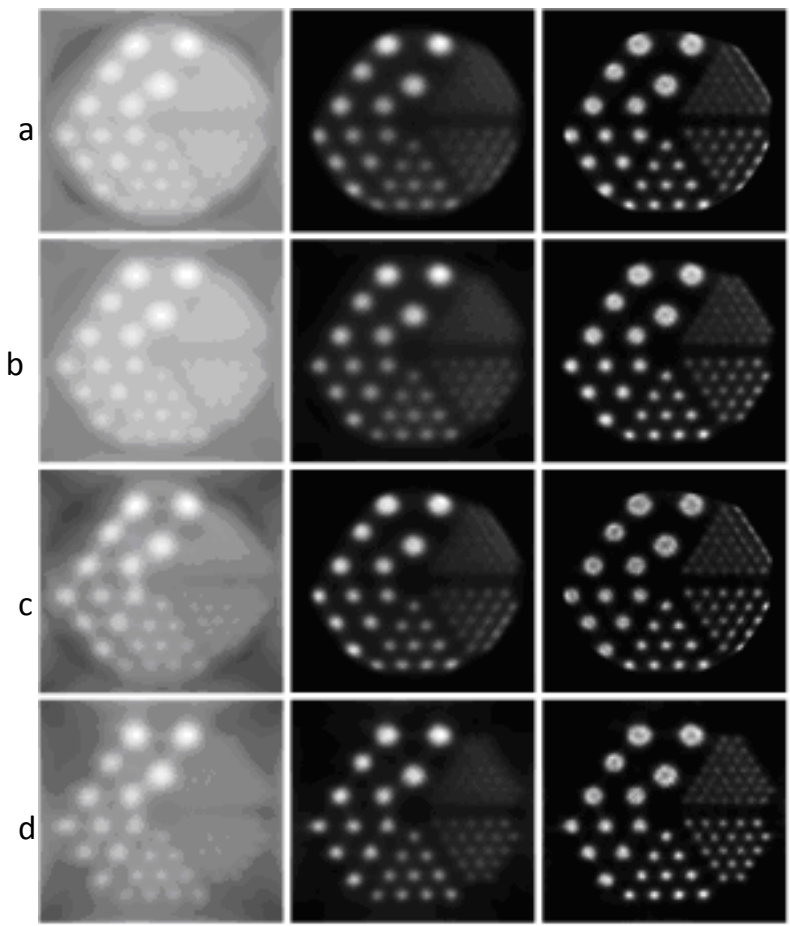

1iter

10iter

50iter

Figure 1. Reconstructed images with: (a) MRP-EMML, (b) MRP-ISRA; (c) MRP-WLS, and d) MRP-ISWLS, after 1, 10 and 50 iterations respectively.

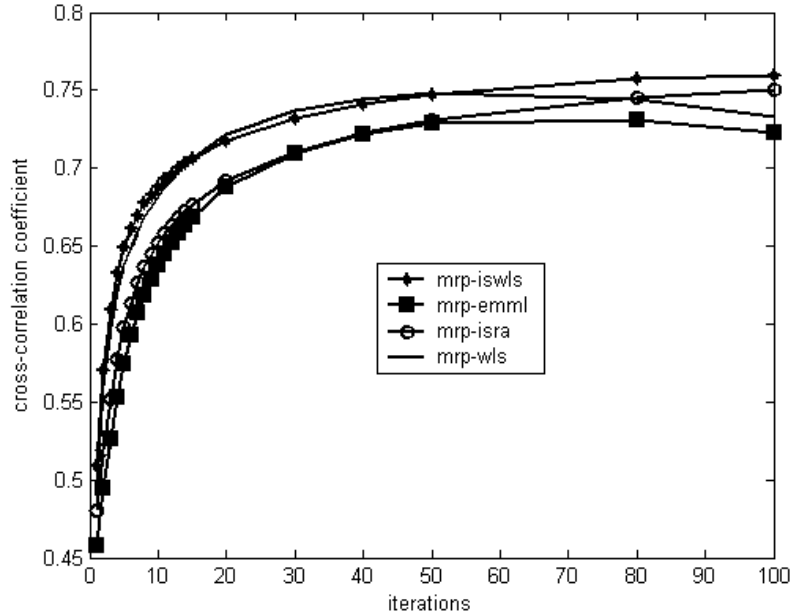

Figure 2. Cross-correlation coefficient versus the number of iterations for MRP-EMML, MRP-ISRA, MRP-WLS, and MRP-ISWLS.

measure between reconstructed and real radio distribution image. Its values are in the range $[-1,1]$. Value $c=1$ corresponds to fully correlated images.

Except for the cross-correlation coefficient that shows the average performance of the reconstruction methods, local contrast-to-noise ratios (CNR) [7] for rods with different diameters were calculated. CNRs for 4.8, 3.2, and $1.6 \mathrm{~mm}$ rods diameter were computed, using squared regions-of-interest (ROIs), 4.55, 3.85 and $2.15 \mathrm{~mm}$ in size, respectively. The ROIs were placed inside the corresponding objects. The number of selected ROIs was equal to the number of same sized objects. ROIs of the same sizes were positioned in three different background areas, each. $C N R_{R O I}$ was defined as:

$$
C N R_{R O I}=\frac{R_{O b j_{R O I}}-R_{B a c k g} g_{R O I}}{\sigma_{\text {Backg }_{R O I}}},
$$

where $R_{o b j_{R O I}}$ is the mean value of reconstructed objects in the corresponding ROIs, and $R_{\text {Backg }}$ ioI is the mean value of the three background ROIs in each case. Further, $\sigma_{\text {Backg }_{\text {ROI }}}$ is the standard deviation of background values in the corresponding ROIs. The graphs in Figure 3 illustrate the variation of $\mathrm{CNR}_{\mathrm{ROI}}$ with respect to the number of iterations for the three different object diameters.

\section{DISCUSSION}

MRP-ISWLS presents higher cross-correlation values than MRP-EM-ML and MRP-ISRA. Although it shows the same high values of cross-correlation coefficient as MRP-WLS during the first 50 iterations it has better noise manipulation. In our research cross-correlation coefficient reaches up to 0.75 , and not to 1 . This is because extra corrections prior or during the reconstruction should be made, like attenuation, scatter and random 

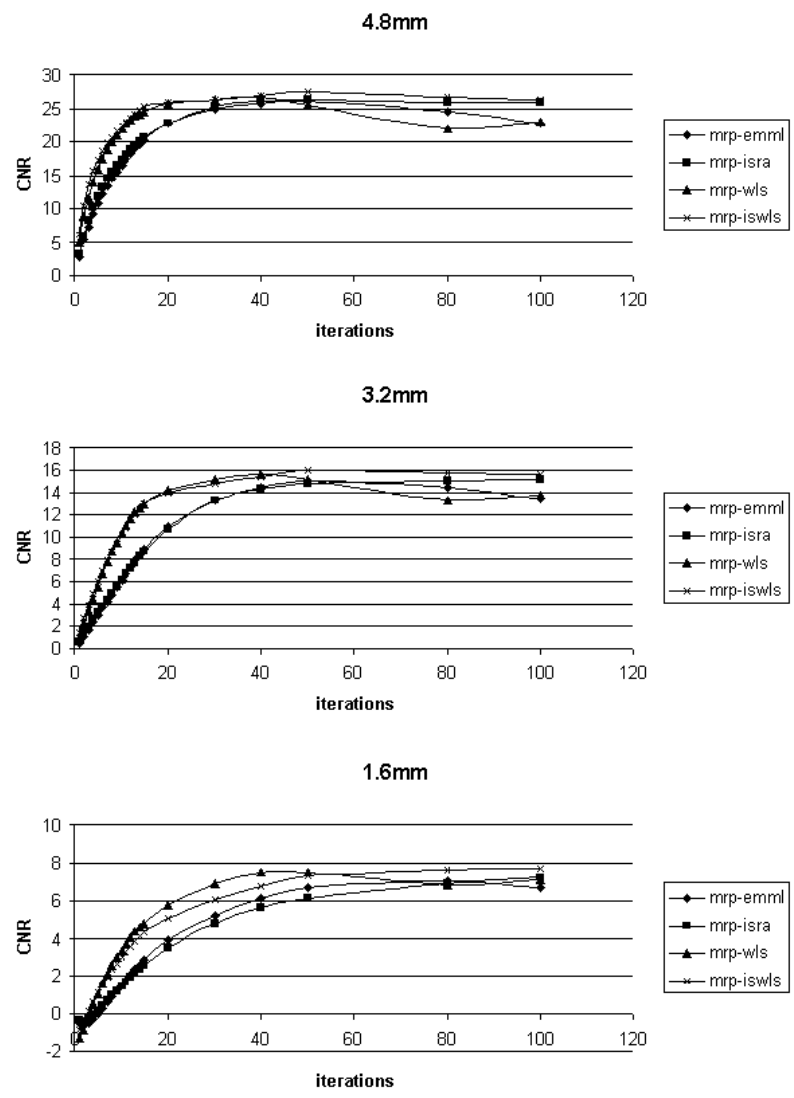

Figure 3. CNRs versus iterations for: (a) $4.8 \mathrm{~mm}$; (b) $3.2 \mathrm{~mm}$ and (c) $1.6 \mathrm{~mm}$ object diameter.

corrections. Despite the fact that these corrections have not been made the final result of the average performance of MRP-ISWLS is not altered.

As illustrated in Figure 3 MRP-ISWLS presents high CNR ratios from the first iterations, higher than MRPEM-ML and MRP-ISRA. Although it shows similar performance to MRP-WLS, its CNR ratios do not degrade after 50 iterations but tend to be stabilized. So, MRPISWLS presents a better noise manipulation than MRPWLS.

In order to determine a satisfactory value of $b$, various values were applied to MRP-ISWLS. These values were $0.001,0.01,0.1,1,1.5$. The value $b=0.001$ presented higher CNR values in comparison to ISWLS's results.

MRP-ISRA and MRP_ISWLS are slower than MRPEM-ML and MRP-WLS during the first 9 iterations (79 s/iteration). Their reconstruction speed is gradually improving with increasing number of iterations. The reason for slow reconstruction process during the first iterations lies in the time needed for backprojection computations in the first iteration.

\section{CONCLUSION}

In this work, different simultaneous iterative reconstruction schemes were applied to data acquired from a simulation of a small-animal PET scanner. A new iterative scheme was introduced, namely MRP-ISWLS.

\section{ACKNOWLEDGEMENTS}

This research has been co-financed by the European Union (European Social Fund-ESF) and Greek national funds through the Operational Program "Education and Lifelong Learning” of the National Strategic Reference Framework (NSRF)-Research Funding Program: THALES: Reinforcement of the interdisciplinary and/or inter-institutional research and innovation.

\section{REFERENCES}

[1] Phelps M.E. (2004) PET Molecular Imaging and its Applications. Chapter 1, Springer-Verlag, New York.

[2] Daube-Witherspoon, M.E. and Muehllehner, G. (1986) An iterative image space reconstruction algorithm suitable for volume ECT. IEEE Transactions on Medical Imaging, 5, 61-66.

[3] Anderson, M.M., Mair, B.A., Rao, M. and Wu, C.H. (1997) Weighted least-squares reconstruction methods for positron emission tomography. IEEE Transactions on Medical Imaging, 16, 159-165. http://dx.doi.org/10.1109/42.563661

[4] Alenius, S. Ruotsalainen, U. and Astola J. (1997) Using local median as the location of the prior distribution in iterative emission tomography image reconstruction. Proceedings of IEEE Medical Image Conference, 45, 30973104.

[5] Karali, E., Pavlopoulos, S., Lambropoulou, S. and Koutsouris, D. (2011). ISWLS: A novel algorithm for image reconstruction in PET. IEEE Transactions on Information Technology in Biomedicine, 15, 381-386. http://dx.doi.org/10.1109/TITB.2010.2104161

[6] Green, P.J. (1990) On use of the EM algorithm for penalized likelihood estimation. Journal of Royal Statistical Society Series B, 52, 443-452.

[7] Cherry, S.R., Sorenson, J.A. and Phelps M.E. (2003) Physics in Nuclear Medicine. Chapter 15, SAUNDERSElsevier, Philadelphia. 